\title{
On the Doppler navigation of a small body orbiter: Rosetta
}

\author{
E. Mysen ${ }^{1,2}$ and K. Aksnes ${ }^{1}$ \\ 1 Institute of Theoretical Astrophysics, University of Oslo, PO Box 1029 Blindern, 0315 Oslo, Norway \\ e-mail: eirik.mysen@astro.uio.no \\ 2 Visiting Scientist, Global Aerospace Corporation, 711 W. Woodbury Road, Suite H, Altadena, CA 91001-5327, USA
}

Received 10 April 2008 / Accepted 27 August 2008

\section{ABSTRACT}

\begin{abstract}
In 2014 the ESA probe Rosetta is to rendezvous with short-period comet 67P/Churyumov-Gerasimenko, where it will be inserted into a stable bound orbit. Partly by measuring the Doppler displacements of the probe's radio carrier waves, the gravity field of the comet's nucleus will be found. In this work, navigation strategies are developed, and important error sources for and feasibility of the comet nucleus mass determination are presented.
\end{abstract}

Key words. space vehicles - comets: individual: 67P/Churyumov-Gerasimenko - methods: numerical - methods: analytical

\section{Introduction}

The navigation of probes in deep space is mainly accomplished by processing information delivered by the spacecraft's radio carrier waves, in the equivalents of probe distance (range) and line-of-sight velocity (range-rate). The processing itself consists of iteratively finding the system parameters, of an assumed model for the spacecraft motion, which are consistent with the observed data. Thus, one obtains not only the probe's position and velocity, but also scientifically interesting quantities like the mass and details of the gravity field of celestial bodies perturbing the spacecraft.

This is exactly one of the subgoals of space missions like Rosetta: the gravity field inversion of comet 67P/ChuryumovGerasimenko by the Rosetta probe in a bound orbit around the comet's nucleus. In this work, the content of the range-rate data from a cometary orbiter is discussed. The focus of this study is on the determination of the nucleus mass, and the spacecraft's position and velocity relative to the comet.

\section{The comet nucleus}

Some plausible values for the nucleus mass $m_{\mathrm{c}}$ of target comet 67P/Churyumov-Gerasimenko are

$0.1($ low $)-1($ nominal $)-2$ (high) $\times 10^{13} \mathrm{~kg}$

based on Rickman et al. (1987), Mysen (2004), Davidsson \& Gutiérrez (2005) and Kossacki \& Szutowicz (2006). As a unit for length, we have found it convenient to use what we here define as the mean nucleus radius $r_{\mathrm{c}} \equiv 2 \mathrm{~km}$, which can be compared to the effective radius (that of the equivalent sphere) of Lamy et al. (2007): $1.72 \mathrm{~km}$. For the product of the constant of gravitation and nominal nucleus mass above, we then have $\mu_{\mathrm{c}}=1.08 r_{\mathrm{c}}^{3} \mathrm{~h}^{-2}$ ( $\mathrm{h} \equiv$ hour), used throughout this work.

\section{Observable}

\subsection{Simplification}

Range observations $\varrho$ are according to Weeks (1995) useful in determining the comet ephemeris, but only marginally informative for the estimation of the cometocentric probe orbit. Not taking in to account imaging, leaves us with the range-rate observations $\dot{\varrho}$.

In reality, the range-rate, or Doppler observable, is a measurement of the number of radio carrier wave cycles accumulated by a receiver relative to a reference signal, divided by the accumulation time $\Delta t$ (Moyer 2003). If we set this observable equal to the probe's line-of-sight velocity, one then assumes that the accumulation rate of cycles is constant during the planned count time $\Delta t \approx 1000 \mathrm{~s}$ of the Rosetta orbiter phase. In this work, with its focus on the comet nucleus mass determination, we are mainly interested in the range-rate variation with period equal to the cometocentric orbit period of the spacecraft. The integration time $\Delta t$ is therefore much shorter than the timescale on which the the relevant range-rate component changes, and, as a result, our assumption is fair.

With the integration time $\Delta t \approx 1000 \mathrm{~s}$, the standard deviation of the assumed Gaussian white noise instrumental observation error is $\delta \dot{\varrho} \approx 0.01 \mathrm{~mm} \mathrm{~s}^{-1}$ (Pätzold et al. 2007).

\subsection{Formulation}

Let the inertially fixed $z$-axis of our cometocentric reference frame coincide with the direction of the mass center of the Earth at the time of the first data point, $t=t_{0}=0$. Also, let the inertially fixed $x$-axis be defined by the direction of the comet's geocentric apparent (non-radial) motion at the start of the tracking pass. By definition, this reference system does not rotate relative to an inertial one. Finally, let the geocentric position of the comet be given by $\boldsymbol{R}$, the cometocentric position of the spacecraft by $\boldsymbol{r}$, and the geocentric position of the tracking station by $\boldsymbol{R}_{\oplus}$. 


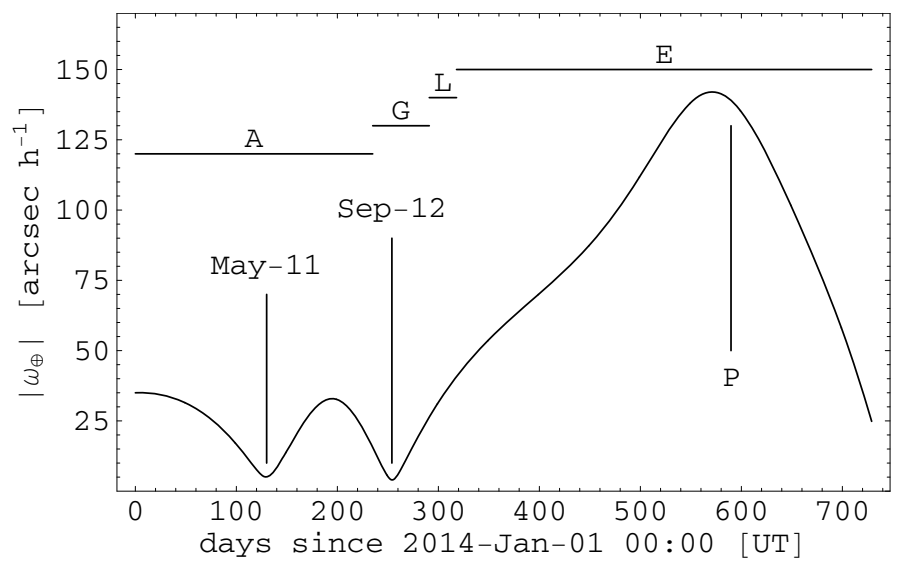

Fig. 1. The figure shows the angular velocity (apparent motion) of the target comet as seen from Earth during the comet rendezvous and orbiter phase years of Rosetta, 2014-2015. A): comet approach phase, G): gravity campaign, L): lander delivery to nucleus, E): comet escort phase, $\mathbf{P}$ ): perihelion passage.

Now, we are interested in the Doppler components that contain information on the cometocentric orbit and nucleus mass, i.e., the components of the range-rate that are dependent on $\boldsymbol{r}$ or $\dot{r}$ only:

$$
\begin{aligned}
\dot{\varrho}(\boldsymbol{r}, \dot{\boldsymbol{r}})= & \dot{\varrho} \cdot \frac{\varrho}{\varrho}=\dot{\boldsymbol{r}} \cdot \boldsymbol{u}_{\mathrm{R}}+\dot{\boldsymbol{R}} \cdot \frac{\boldsymbol{r}}{R} \\
& -\left(\dot{\boldsymbol{R}} \cdot \boldsymbol{u}_{\mathrm{R}}\right)\left(\frac{\boldsymbol{r}}{R} \cdot \boldsymbol{u}_{\mathrm{R}}\right)+O\left(\dot{\boldsymbol{R}}_{\oplus} \cdot \frac{\boldsymbol{r}}{R}\right)
\end{aligned}
$$

where $\boldsymbol{u}_{\mathrm{R}} \equiv \boldsymbol{R} / R$ and all terms not dependent on $\boldsymbol{r}$ or $\dot{\boldsymbol{r}}$ have been removed. This expression can be further simplified if the motion of the comet mass center is written as

$$
\begin{aligned}
\boldsymbol{R} & =\boldsymbol{R}\left(t_{0}\right)+\dot{\boldsymbol{R}}\left(t_{0}\right) t+\frac{1}{2} \boldsymbol{A}\left(t_{0}\right) t^{2} \\
& =-R_{0} \boldsymbol{u}_{z}+\left(v_{R} \boldsymbol{u}_{z}+v_{T} \boldsymbol{u}_{x}\right) t+\frac{1}{2} \sum_{i=1}^{3} A_{x_{i}} \boldsymbol{u}_{x_{i}} t^{2}
\end{aligned}
$$

where $\boldsymbol{u}_{x_{i}}$ are unit vectors along the reference system axes, and $R_{0}, v_{T}, v_{R}$ and $A_{x_{i}}$ are constants. After some calculation one obtains for the range-rate

$\dot{\varrho}(\boldsymbol{r}, \dot{\boldsymbol{r}}) \approx-\dot{z}+\omega_{\oplus}(x+\dot{x} t), \quad v_{\mathrm{T}} \equiv \omega_{\oplus} R_{0}$

if the probe is sufficiently close to the comet, $|\boldsymbol{r}| \lesssim 50 r_{\mathrm{c}}$. Approaches similar to Eq. (4) are given in Wood (1986) and Russell \& Thurman (1989). In what follows, the range-rate observable will simply be defined by Eq. (4), which will demonstrate the essential challenges of the orbit and mass determination.

\subsection{Degeneracies}

Included as Fig. 1 is a plot of $\left|\omega_{\oplus}\right|$ for the target comet during the orbiter phase years 2014-2015. The values are produced by JPL's HORIZONS with

$\left|\omega_{\oplus}\right|=\sqrt{\dot{\alpha_{\oplus}^{2} \cos ^{2} \delta_{\oplus}+{\dot{\delta_{\oplus}}}^{2}}}$

where $\delta_{\oplus}$ and $\alpha_{\oplus}$ are the declination and right ascension of the comet relative to the Earth.

If one then assumes that $\dot{x}$ and $\dot{z}$ are roughly of the same order of magnitude and use a mean $\omega_{\oplus}=20 \operatorname{arcsech}^{-1}$ for 2014,

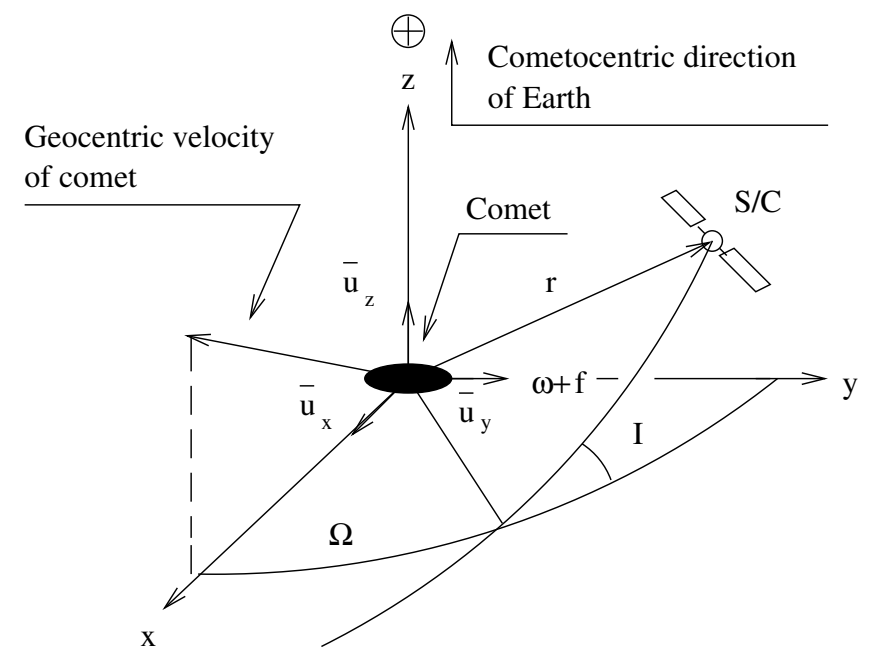

Fig. 2. The cometocentric non-rotating reference system is shown at the start of the tracking pass $t_{0}$, when the $z$-axis points towards Earth. Note that the comet's geocentric velocity at $t_{0}$ by definition lies in the $x z$-plane. The unit vectors along the reference axes are given by $\boldsymbol{u}_{x_{i}}$.

the contribution to the range-rate observable Eq. (4) associated with the $\dot{x}$ term equals that of $\dot{z}$ after a very long time $t \sim 10^{4} \mathrm{~h}$. However, one can write $z$ and $\dot{z}$ in terms of the probe's cometocentric orbit elements

$z=\frac{a\left(1-e^{2}\right)}{1+e \cos f} \sin I \sin (\omega+f)$

$\dot{z}=\frac{n a \sin I[e \cos \omega+\cos (\omega+f)]}{\sqrt{1-e^{2}}}$.

Here $a$ is the semi-major axis of the probe's cometocentric orbit, $e$ the eccentricity, $\omega$ the argument of pericenter and $I$ the inclination of the orbit with respect to the cometocentric reference system (see Fig. 2).

For instance, $I=0$ means that the orbit lies normal to the geocentric line-of-sight at $t=t_{0}$. The true anomaly is given by $f$ and $n \equiv \sqrt{\mu_{\mathrm{c}} / a^{3}}$. Now, basic expressions for the two-body problem yield that $f=f\left(e, n t+M_{0}\right)$ with $M_{0}$ as the value of the mean anomaly at the start of the tracking pass. Consequently, it can be seen from Eq. (7) that there is only information on the parameters

$n, \quad p \equiv a \sin I, \quad e, \quad \omega, \quad M_{0}$

from a Keplerian variation of the $\dot{z}$ component. Equation (6) shows that the inclusion of range observations does not alter this fact. Note that in a Keplerian $\dot{z}$, there is no information on the orbit's longitude of ascending node $\Omega$ either, measured in the observer's initial plane-of-sky.

These well-known properties of the orbiter inversion problem (Wood 1986) means that for $\omega_{\oplus}=0$ and two-body motion, there is no information in the range and range-rate data on the mass of the central body, or on the cometocentric position and velocity of the spacecraft, except for what is directly measurable: $\dot{z}_{0}$. So, unless the Keplerian motion is strongly perturbed, the determination of comet nucleus mass and probe orbit are actually entirely dependent on the $x$ and $\dot{x}$ terms of the observable Eq. (4). For instance, if $\dot{x}$ carries information on $I$, then $a$ is determined from $p$ which is easily available from $\dot{z}$. Together with $n$, which is also well determined from $\dot{z}$, the nucleus mass is given. 


\section{Estimation by Kalman filter}

\subsection{Numerical}

One simple way to find values of unknown parameters affecting the spacecraft's motion from the range-rate observations, Eq. (4), is to employ a first-order linearized Kalman filter (Gelb 1974). A Kalman filter takes an observation, compares it with a value of this observation computed from an initial guess of parameter values, and maps the difference in to parameter corrections. These corrected parameters then serve as better guesses for the next iteration. The precisions of and correlations between the parameters $\theta_{i}$ to be evaluated are then given by the covariance matrix $S_{i j}$. For a range-rate $\dot{\varrho}$ observation with $\delta \dot{\varrho}$ as a one-sigma noise level, the inverse of the matrix $S$ is changed according to

$\Delta S_{i j}^{-1}=\frac{I_{i j}}{(\delta \dot{\varrho})^{2}}, \quad I_{i j}=\frac{\partial \dot{\varrho}}{\partial \theta i} \frac{\partial \dot{\varrho}}{\partial \theta j}$,

yielding the square of the parameter precisions $S_{i i}=\left(\delta \theta_{i}\right)^{2}$, the expected deviation of the filter estimate of the parameter from the parameter's true value. The correlation between parameters $\theta_{i}$ and $\theta_{j}$ is given by

$\operatorname{corr}_{\left(\theta_{i}, \theta_{j}\right)} \equiv \frac{S_{i j}}{\sqrt{S_{i i} S_{j j}}}, \quad\left|\operatorname{corr}_{\left(\theta_{i}, \theta_{j}\right)}\right| \leq 1$

and is a measure of the extent to which the filter can separate the parameters relative to their current estimated precisions.

Equation (9) forms the basis for simulations of parameter precisions. In these calculations we will obtain the partial derivatives of the observation $\dot{\varrho}$ with respect to each parameter by solving a coupled set of differential equations along the a priori, or initial guess, trajectory, also called variational equations. For instance, the partial derivative of the range-rate Eq. (4) with respect to the nucleus mass

$$
\begin{aligned}
\frac{\partial \dot{\underline{\varrho}}}{\partial \mu_{\mathrm{c}}} & =-\frac{\partial \dot{z}}{\partial \mu_{\mathrm{c}}}+\omega_{\oplus}\left[\frac{\partial x}{\partial \mu_{\mathrm{c}}}+\frac{\partial \dot{x}}{\partial \mu_{\mathrm{c}}} t\right] \\
& =-\frac{\mathrm{d}}{\mathrm{d} t}\left(\frac{\partial z}{\partial \mu_{\mathrm{c}}}\right)+\omega_{\oplus}\left[\frac{\partial x}{\partial \mu_{\mathrm{c}}}+t \frac{\mathrm{d}}{\mathrm{d} t}\left(\frac{\partial x}{\partial \mu_{\mathrm{c}}}\right)\right],
\end{aligned}
$$

which is necessary for Eq. (9), can be obtained from the three coupled second-order differential equations

$$
\frac{\mathrm{d}^{2}}{\mathrm{~d} t^{2}}\left(\frac{\partial x_{i}}{\partial \mu_{\mathrm{c}}}\right)=\sum_{j=1}^{3} \frac{\partial \ddot{x}_{i}}{\partial x_{j}}\left(\frac{\partial x_{j}}{\partial \mu_{\mathrm{c}}}\right)+\frac{\partial \ddot{x}_{i}}{\partial \mu_{\mathrm{c}}}, \quad i=1,2,3 .
$$

The non-bracketed terms above are known functions of time from the a priori trajectory, where the last term is due to the acceleration's explicit dependency on mass, $\ddot{x}=\ddot{x}\left[\mu_{\mathrm{c}}, x\left(\mu_{\mathrm{c}}\right)\right]$. zation

Previously, we have shown, with the use of the parameteri-

$\dot{z}=\dot{z}\left(n, p, e, \omega, M_{0}\right)$,

that a Keplerian variation of $\dot{z}$ does not contain information on the nucleus mass since $\partial \dot{z} / \partial \mu_{\mathrm{c}}=0$. Here, it should be pointed out that a solution $\partial \dot{z} / \partial \mu_{\mathrm{c}} \neq 0$ of Eq. (12) does not necessarily mean that there is information on the nucleus mass in the variation of $\dot{z}$. For instance, if we use the parameterization

$\dot{z}=\dot{z}\left(\mu_{\mathrm{c}}, \boldsymbol{r}_{0}, \dot{\boldsymbol{r}}_{0}\right), \quad \boldsymbol{r}_{0} \equiv \boldsymbol{r}\left(t_{0}\right) \quad$ etc.

for Eq. (12), simulations yield that $\partial \dot{z} / \partial \mu_{\mathrm{c}} \neq 0$ even though the motion is defined to be strictly Keplerian. However, substitution of the simulations into Eq. (9) reveals that there is no improvement in the estimated precision of the mass as observations are processed, i.e., there is no information on the nucleus mass. That is, the non-zero partial is in this case merely the result of a particular parameterization, and the previously stated degeneracies are recovered by the filter Eq. (9).

\subsection{Analytical}

If analytical expressions for the precisions are sought, orbital elements will be used to describe the spacecraft's state relative to the comet, together with the continuous version of Eq. (9), namely (see for instance Anderson \& Giampieri 1999)

$S_{i j}^{-1}\left(t_{1}\right)-S_{i j}^{-1}\left(t_{0}\right)=\frac{1}{(\delta \dot{\varrho})^{2}} \frac{\bar{I}_{i j}}{\Delta t}, \quad \bar{I}_{i j}=\int_{0}^{T} \mathrm{~d} t I_{i j}$.

In this equation, which is a version of the Riccati equation without process noise (Gelb 1974), the time of the first data point is $t=t_{0}=0$, and the length of the tracking pass is $T$. Analytic expressions for the parameter precisions can then be obtained by inverting the matrix $S^{-1}$ above. Although inversions of analytic matrices are possible with modern computers and algebraic software, the resulting expressions are cumbersome, and therefore of limited use. We will therefore assume that the correlations of the system are small, representing a best-case scenario. That is, the off-diagonal elements of $S^{-1}$ are small so that the precisions are well represented by the diagonal elements. If there, in addition to small correlations, is no a priori information on the parameters, Eq. (15) is reduced to

$\left(\frac{\delta \dot{\underline{\varrho}}}{\delta \theta_{i}}\right)^{2}=\frac{1}{\Delta t} \int_{0}^{T} \mathrm{~d} t\left(\frac{\partial \dot{\underline{\varrho}}}{\partial \theta_{i}}\right)^{2}$

with $\Delta t$ still as the time between data points.

\section{Mass determination due to relative motion}

\subsection{Optimal inversion}

Usually in deep space navigation, most of the information on the mass of the central body and the motion of the spacecraft is given by the relative motion of the observer's cometocentric planeof-sky. To be more specific, let the $x_{i}$ of Eq. (4) be Keplerian, with the result that the cometocentric orbit elements are constant. As previously mentioned, there is no information on the probe orbit's longitude of ascending node $\Omega$ in the observer's initial plane-of-sky, or on the mass parameter $\mu_{\mathrm{c}}$ in a Keplerian $\dot{z}$. This is not the case, however, for the other components of Eq. (4). For instance

$\dot{x}=\dot{x}\left[a\left(n, \mu_{\mathrm{c}}\right), I\left(p, n, \mu_{\mathrm{c}}\right), \Omega\right]$

which can be found in most textbooks in celestial mechanics. It should be noted that with the parameterization above, we have that both

$\frac{\partial \dot{z}}{\partial \mu_{\mathrm{c}}}=\frac{\partial \dot{z}}{\partial \Omega}=0$

That is, the fact that a Keplerian variation of $\dot{z}$ does not contain information on the nucleus mass has been resolved already at the parameterization level. Taking the necessary partial derivatives of Eq. (4) using the dependency of Eq. (17), one gets to zero order in orbit eccentricity

$$
\int_{0}^{T} \mathrm{~d} t\left(\frac{\partial \underline{\underline{e}}}{\partial \Omega}\right)^{2} \rightarrow \omega_{\oplus}^{2} \frac{n^{2} a^{2} T^{3}}{6}\left(1-\cos ^{2} \Omega \sin ^{2} I\right)
$$


and

$\int_{0}^{T} \mathrm{~d} t\left(\frac{\partial \dot{\underline{\varrho}}}{\partial \mu_{\mathrm{c}}}\right)^{2} \rightarrow \omega_{\oplus}^{2} \frac{n^{2} a^{2} T^{3}}{54 \mu_{\mathrm{c}}^{2}} \frac{1-\cos ^{2} \Omega \sin ^{2} I}{\cos ^{2} I}$

as $T \rightarrow \infty$. With the use of Eq. (16) we then get

$(\delta \Omega)^{2}=\left(\frac{\delta \dot{\varrho}}{a \omega_{\oplus}}\right)^{2} \frac{\Delta t}{n^{2} T^{3}} \frac{6}{1-\cos ^{2} \Omega \sin ^{2} I}$

and

$$
\left(\frac{\delta \mu_{\mathrm{c}}}{\mu_{\mathrm{c}}}\right)^{2}=\left(\frac{\delta m_{\mathrm{c}}}{m_{\mathrm{c}}}\right)^{2}=9(\delta \Omega)^{2} \cos ^{2} I
$$

for the precision of the node and nucleus mass as estimated by the Kalman filter Eq. (9).

These expressions should be taken as the best possible results produced by Eq. (9) since a prerequisite of Eq. (16) is that the parameter correlations are low. As we shall see, the Kalman filter Eq. (9) produces precisions that are not as good as those of Eqs. (21) and (22).

A quantitative approach like above is also given by Russell \& Thurman (1989) in which no restrictive assumptions on parameter correlations are made. However, the mass of the central body is assumed to be known, and the resulting expressions for the remaining parameter precisions are given indirectly only, due to their lengths. In addition, some erroneous results are obtained, as pointed out in their work. The errors are related to the fact that in their treatment, the partial derivatives of the observable with respect to solve-for parameters are determined by the largest assumed non-zero component of Eq. (4) only. If these partials vanish for some orbit geometry, one is then perhaps falsely lead to believe that there is a singularity in the data. Also, only the average information in an orbit is considered in their work.

\subsection{Simulations of covariance}

Next, we want compare the analytic expression Eq. (22) to covariance simulations Eq. (9) using Eq. (4) as the Doppler observable. In order to generate the covariance matrix of Eq. (9), the variational equations Eq. (12) are simulated along the studied trajectory. It should be stressed that such covariance simulations are not dependent on the actual observations and, therefore, we do not need to generate "noisy" data for this purpose. The unknown parameters, of the defined Keplerian motion, to be estimated by the Kalman filter are

$\boldsymbol{r}_{0}, \quad \dot{\boldsymbol{r}}_{0}, \quad \mu_{\mathrm{c}}$.

In Fig. 3 the analytic Eq. (22) is represented by the solid lines, while the outputs of the Kalman filter are given by the dashed curves, where the mass of the nucleus is set to our nominal value, Eq. (1), and the longitude of ascending node is set to an arbitrary $\Omega=1.00$. Due to the asymptotic prerequisite $T \rightarrow \infty$ of Eq. (22), we do not expect the expression to capture properties of the Kalman filter output for tracking intervals $T$ less than an orbit period $P$. Therefore the analytic (solid) curves have not been plotted for $T<P$. The curves in the plot are separated by the value of the orbit inclination $I$ with respect to the observer's initial plane-of-sky, as measured in radians.

As can be seen, the correspondence is acceptable considering the assumptions. It should be pointed out that the choice of a non-zero eccentricity $e=0.2$ is not by chance, but represents a value for which the correlations of the parameters drop from

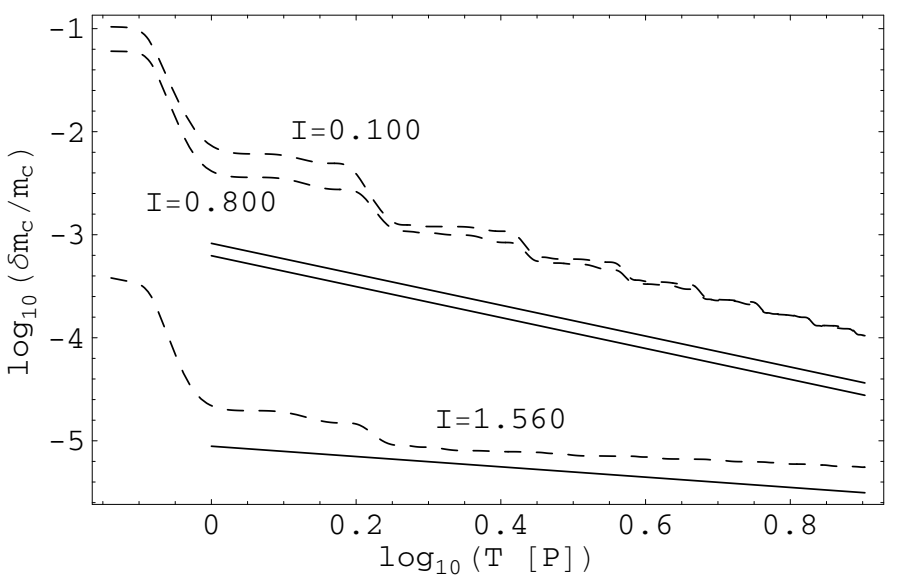

Fig. 3. Mass precision estimates are shown as functions of the tracking time $T$ in the number of orbit periods $P=191 \mathrm{~h}$ for a cometocentric orbit semi-major axis of $a=10 r_{\mathrm{c}}$ of the spacecraft. The numerical simulations (dashed lines) Eq. (9) are compared with analytical results (solid lines) from Eq. (22) $(I=0.100,0.800 \mathrm{rad}$.) and Eq. (26) $(I=$ $1.560 \mathrm{rad}$.), with $\omega_{\oplus}=20 \operatorname{arcsec~h}^{-1}$.

very high to high. That circular orbits should be avoided has been pointed out in previous works, see for instance Russell \& Thurman (1989).

Figure 3 reveals a decorrelation timescale, i.e., the timescale on which the precisions converge to their asymptotic tendencies, of about an orbit period. If we include more unknown parameters in addition to those of Eq. (23), the Kalman filter will have a harder time separating the parameters, and the decorrelation timescale will increase.

At $I=1.560$, not set to $\pi / 2$ in order to avoid purely mathematical results, the simulated points drop roughly as $\sim T^{-1 / 2}$ in disagreement with Eq. (22) whose neglected components will then dominate. That is, the information in the Doppler data on the position and velocity of the spacecraft relative to the comet is so abundant that the probe's state is, for our purposes, almost completely known. To elaborate, we can use Kepler's third law in the form

$\mu_{\mathrm{c}}=n^{2} p^{3} \sin ^{-3} I, \quad p \equiv a \sin I$.

Now, from Eq. (7) it can be reasoned that if $I \neq 0$

$\frac{\partial \dot{e}}{\partial n} \sim \frac{\partial \dot{z}}{\partial n} \sim t \sin n t$

and we expect that $\delta n \sim T^{-3 / 2}$, see Eq. (16). That is, the uncertainty in $n$ is rapidly reduced. Then we have the inclination component which is determined if the hypothesis of high precision values of $\boldsymbol{r}_{0}$ and $\dot{\boldsymbol{r}}_{0}$ hold. This leaves us with the parameter $p$ which, from a Keplerian $\dot{z}$ of Eq. (4) and simplification of Eq. (16), has an estimated precision

$\left(\frac{\delta \mu_{\mathrm{c}}}{\mu_{\mathrm{c}}}\right)^{2} \sim\left(\frac{3 \delta p}{p}\right)^{2} \sim 18 \frac{a}{\mu_{\mathrm{c}}} \frac{\Delta t}{T}\left(\frac{\delta \dot{\varrho}}{\sin I}\right)^{2}$.

This equation is also represented in Fig. 3 with $I=1.560$, and again the match with the simulation is acceptable.

\subsection{Loss of signal component}

According to Fig. 3, the precision of the mass estimate does not degrade significantly when the orbit gets more aligned with the 
observer's initial plane-of-sky, $I \rightarrow 0$. On the contrary, simulations with a nominal nucleus mass show that even circular orbits yield good precisions for this geometry. This is of some interest as one is led to believe that an $I \neq 0$ strategy is wise because the then non-zero $\dot{z}$ component of the Doppler observable, Eq. (4), might carry information that lowers the parameter correlations.

Unlike what is indicated by Eq. (21), filters in which we solve for the orbit elements yield $\delta \Omega \rightarrow \infty$ as $I \rightarrow 0$. However, filters in which we solve for the spacecraft's position and velocity relative to the nucleus demonstrate that $\boldsymbol{r}_{0}$ and $\dot{\boldsymbol{r}}_{0}$ are still well determined for such orbits. The reason for this apparent inconsistency is the perfect correlation between $\Omega$ and $\omega$ which occurs when $I \rightarrow 0$. That is, for $I=0$ the observable Eq. (4) is dependent on $\Omega+\omega$ only.

\subsection{A singularity}

At last, it should be pointed out that as $I \rightarrow \pi / 2$ when $\Omega=0$ or $\pi$, the precision of the node, Eq. (21), goes towards infinity. This well-known unfavourable geometry, referred to in literature as a singularity in the Doppler data, means that the information on the spacecraft's position and velocity relative to the comet is seriously degraded. That is, as this orbit configuration is approached, then

$\delta \dot{y}\left(t_{0}\right) \sim \frac{\partial \dot{y}\left(t_{0}\right)}{\partial \Omega} \times \delta \Omega \sim 1 \times \frac{1}{\cos I} \sim \infty \sim \delta y\left(t_{0}\right)$,

but

$\delta \dot{x}\left(t_{0}\right) \sim \frac{\partial \dot{x}\left(t_{0}\right)}{\partial \Omega} \times \delta \Omega \sim \cos I \times \frac{1}{\cos I} \sim 1 \sim \delta x\left(t_{0}\right)$.

As for the mass precision estimate, Eq. (22) indicates that there is no such singularity, as is confirmed by covariance simulations.

In Wood (1986) the singularities of the navigation and, indirectly, also the mass inversion problem have been pointed out in an approach where the average information in an orbit is studied. In this paper, we have also attempted to formulate expressions which capture not only the qualitative, but also to some extent the quantitative aspects of the problem. This enables us to better compare the role of the different parameters in the navigation of the probe and determination of the nucleus mass, but elements which are related to extreme parameter correlations, i.e. circular orbits, are not captured in the analytical formulation of this paper.

\section{Perturbations}

As previously mentioned, if the motion of the orbiter is Keplerian, one is, from Doppler observations alone, reliant on the rotation of the observer's plane-of-sky if the spacecraft's state relative to the central body and the body's mass are to be determined. However, the Rosetta probe, with its large solar cell arrays, will experience strong non-gravitational perturbations both due to radiation pressure from the Sun and, near the comet nucleus, outgassing pressure. In this section, we will therefore investigate to what extent the relevant perturbations can influence navigation and nucleus mass determination through Doppler measurements.

\subsection{Radiation pressure}

For this purpose, let $\boldsymbol{n}$ be the unit vector normal to Rosetta's radiation exposed solar cell arrays. By intent the arrays are built to absorb most of the photons, which yields a cometocentric perturbing acceleration acting in the anti-solar direction. If we set the weaker accelerations due to specular and diffuse reflection of solar radiation (Valorge 1995) equal to zero, then one has for the perturbing cometocentric acceleration

$\Delta \ddot{\boldsymbol{r}}=-\frac{\xi}{R^{2}} \chi_{\odot} \boldsymbol{u}_{\odot}$

Above, $\boldsymbol{u}_{\odot}$ is a unit vector pointing in the direction of the Sun, and $\chi_{\odot} \equiv\left|\boldsymbol{n} \cdot \boldsymbol{u}_{\odot}\right|$. If all photons are absorbed and the fuel spent, a large value of $\xi \approx 0.0015 r_{\mathrm{c}} \mathrm{h}^{-2} \mathrm{AU}^{2}$ is obtained (remembering that $r_{\mathrm{c}} \equiv 2 \mathrm{~km}$ is merely our chosen unit of length).

\subsection{Outgassing pressure}

One way to describe interaction between the spacecraft and the expanding gas of the nucleus coma is by the perturbing pressure field acceleration

$\Delta \ddot{\boldsymbol{r}}=\Lambda(\boldsymbol{r})\left[\left(\chi_{r}+\eta_{b}\right) \boldsymbol{u}_{r}+\chi_{r}\left(\eta_{s} \chi_{r}+\eta_{d}\right) \boldsymbol{n}\right]$.

Above, $\chi_{r} \equiv\left|\boldsymbol{n} \cdot \boldsymbol{u}_{r}\right|$ where $\boldsymbol{n}$ now is a unit normal vector of the solar cell arrays that point away from the nucleus, with $\boldsymbol{u}_{r} \equiv \boldsymbol{r} / r$. The "absorption" of molecules by the spacecraft bus is represented by $\eta_{b}$, while the contributions from specular and diffuse reflection are represented by $\eta_{s}$ and $\eta_{d}$ respectively. However, the law's validity, i.e. applicability with constant $\eta$ coefficients, seems tenuous. For instance, whether or not the molecules will be thermalized by the spacecraft surface, and the $\eta$ 's therefore are dependent on, among several effects, the temperature of the probe, remains an open issue which possibly only will be answered when the tracking data are available.

Different models of the pressure field strength are available in the form (see for instance Mysen \& Aksnes 2006, and references therein)

$\Lambda=\bar{\Lambda}(\beta, \lambda)\left(\frac{r_{\mathrm{c}}}{r}\right)^{2}$

where $\beta$ is the cometocentric zenith angle of the spacecraft with respect to the Sun, $\cos \beta=\boldsymbol{u}_{\odot} \cdot \boldsymbol{u}_{r}$, and $\lambda$ is the longitude of the probe measured in the solar plane-of-sky through the pressure field center, and relative to the heliocentric orbit plane of the comet. The inverse square dependency on distance from the field center is related to the mass conservation of a stationary outgassing pressure field. However, from realistic simulations of the coma, it is clear that both the strength of the field and its direction are modulated by the nucleus rotation (see for instance Crifo \& Rodionov 1997a, b).

Using a mass flux

$\rho v \sim \exp [s(\cos \beta-1)]$

where $s>0$ is an anisotropy parameter and $Q$ is a strength parameter, mass conservation yields (see Mysen \& Aksnes 2006)

$\bar{\Lambda}=Q \frac{s \exp [s(\cos \beta-1)]}{2 \pi[1-\exp (-2 s)]}$.

Possible high values of $Q$ are (Mysen \& Aksnes 2006)

$Q_{\mathrm{CO}}\left(10^{27} \mathrm{~mol} \mathrm{~s}^{-1}\right) \sim 1 r_{\mathrm{c}} \mathrm{h}^{-2}, \quad$ all $\mathrm{R}$

$Q_{\mathrm{H}_{2} \mathrm{O}}\left(10^{28} \mathrm{~mol} \mathrm{~s}^{-1}\right) \sim 10 r_{\mathrm{c}} \mathrm{h}^{-2}, \quad$ perihelion

for the total production rates of water and $\mathrm{CO}$ given in brackets. The form of the law is motivated by one direction of preference, namely that of the Sun which drives the sublimation of 
ices. Especially for water outgassing, some simulations (Crifo \& Rodionov 1997a) indicate that the field strength can drop more than an order of magnitude from $\beta=0$ to $\beta=\pi / 2$. As for the sublimation of more volatile ices, which are more relevant at comet rendezvous distances, they seem to yield more isotropic pressure fields (Crifo et al. 2003), i.e. $s$ of Eq. (33) is small. Since they are more volatile, they are also to a larger extent influenced by orbital, instead of diurnal, variations in the received sunlight, and some pressure field variation with $\lambda$ is therefore more plausible based on this simplistic argument alone.

We stress that we here present Eq. (30) merely as an example of a law that is used later to produce examples of the outgassing's influence on our results. A systematic study of the outgassing pressure force on the orbiter is in progress.

\subsection{Perturbation of signal}

The perturbations Eqs. (29) and (30) induce a variation in the cometocentric orbit elements which by an observer is mainly experienced through the dominant part of the observable Eq. (4), namely through $\dot{z}$. Now, let $\zeta$ be the vector containing the parameters which are determinable from a Keplerian variation of this dominant part, which are those given by Eq. (8)

$\zeta=\left(n, p, e, \omega, M_{0}\right)$.

According to the perturbation theory, these parameters experience periodic oscillations $\Delta \zeta$ as well as a so-called secular evolution which below is represented by a linear component

$\zeta=\zeta\left(t_{0}\right)+\dot{\zeta}\left(t_{0}\right) t+\Delta \zeta, \quad t_{0}=0$.

Both types of variation may contain information on for instance the nucleus mass

$$
\frac{\partial \dot{\varrho}}{\partial \mu_{\mathrm{c}}} \approx-\frac{\partial \dot{z}}{\partial \mu_{\mathrm{c}}} \approx-\frac{\partial \dot{z}}{\partial \zeta\left(t_{0}\right)}\left[\frac{\partial \dot{\zeta}\left(t_{0}\right)}{\partial \mu_{\mathrm{c}}} t+\frac{\partial \Delta \zeta}{\partial \mu_{\mathrm{c}}}\right] \neq 0
$$

where the fundamental degeneracy of the Keplerian inversion problem is resolved already at the parameterization level, i.e. $\dot{z}\left[\zeta\left(t_{0}\right)\right] \neq \dot{z}\left(\mu_{\mathrm{c}}\right)$, for Eq. (38) to be valid. It can easily be checked that the secular term is most important for information on nucleus mass and spacecraft velocity and position relative to it. The details of the gravity field, on the other hand, induce a very small secular change in $\zeta$, and the periodic $\Delta \zeta$ is therefore of greater importance as long as the oscillations are detectable. Studies of these components require more physical representations of the outgassing pressure field than that of Eq. (33), and will be treated in a subsequent paper.

In the case of the Rosetta probe where the perturbations are very strong, the linear approximation of the secular evolution above quickly fails, and a Taylor representation is more appropriate

$\zeta=\zeta\left(t_{0}\right)+\dot{\zeta}\left(t_{0}\right) t+\frac{1}{2} \ddot{\zeta}\left(t_{0}\right) t^{2}+\ldots$,

inducing a number of different timescales $m$, as in $\delta \mu_{\mathrm{c}} \sim T^{-m / 2}$ from Eq. (16), in the simulated evolution of the estimated parameter precisions.

Also due to the strong non-gravitational perturbations, the existence of bound orbits on long timescales is questionable, and information on mass from perturbations should not be sought at the expense of stable orbit configurations in which the cometocentric orbit elements by definition do not evolve. Especially so since there, as has been shown previously, still is information on nucleus mass and spacecraft position and velocity relative to the comet from relative motion.

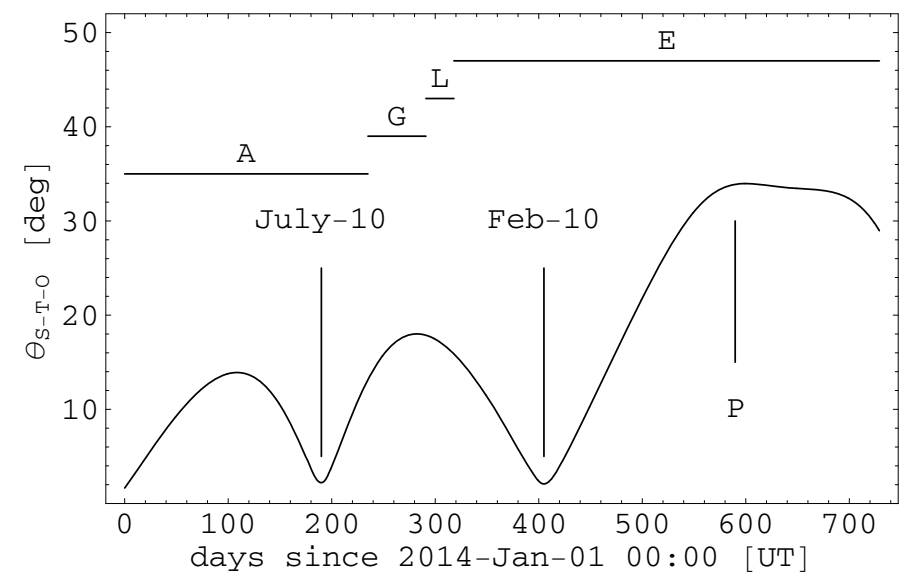

Fig. 4. The figure shows the Sun-comet-Earth angle 2014-2015, with near alignment of Sun-Earth-comet on July 10, 2014 and of Earth-Suncomet on Feb. 10, 2015. A): comet approach phase, G): gravity campaign, L): lander delivery to nucleus, E): comet escort phase, $\mathbf{P}$ ): perihelion passage.

\subsection{Indeterminacy of relative state and mass}

In the same way, information on the node $\Omega$ exists due to the pressure perturbations of the Keplerian signal. However, an exception should be mentioned. Included as Fig. 4 is the angle between cometocentric direction of the Sun and that of the Earth for the rendezvous and orbiter years 2014-2015, based on data from JPL's HORIZONS.

Clearly, this angle is small during the early phases of comet approach and orbit insertion. Let therefore, in order to demonstrate our point, the cometocentric direction of the Sun coincide with that of the Earth. Now, $\zeta$ can be written as a function of canonical variables $\gamma=\left(Q_{\gamma}, P_{\gamma}\right)$ with time derivatives (Tveter 1994; Mysen 2006)

$\dot{Q}_{\gamma} \sim \Delta \ddot{\boldsymbol{r}} \cdot \frac{\partial \boldsymbol{r}}{\partial P_{\gamma}}, \quad \dot{P}_{\gamma} \sim-\Delta \ddot{\boldsymbol{r}} \cdot \frac{\partial \boldsymbol{r}}{\partial Q_{\gamma}}$

when perturbed by the acceleration $\Delta \ddot{\boldsymbol{r}}$. One then has that

$\dot{\zeta} \sim \frac{\partial \zeta}{\partial \gamma} \dot{\gamma} \sim \frac{\partial \zeta}{\partial Q \gamma} \Delta \ddot{z} \frac{\partial z}{\partial P \gamma}-\frac{\partial \zeta}{\partial P \gamma} \Delta \ddot{z} \frac{\partial z}{\partial Q \gamma}$.

Since neither $\zeta, z$, the solar radiation nor the outgassing pressure accelerations $\Delta \ddot{z}$ with angular dependency Eq. (33) contain $\Omega$, the partial derivative of the range-rate with respect to the node, as given by Eq. (38), is zero. That is, if the observer's planeof-sky coincides with the solar plane-of-sky, then the perturbations from the radiation pressure, and types of outgassing pressure with only the Sun as direction of preference, carry no information on the spacecraft's velocity and position relative to the nucleus, as is verified by covariance simulations. The inclusion of derivatives of higher order, like in Eq. (39) does not alter the conclusions.

The same simulations show that if the only effect which carries information on the nucleus mass is an outgassing pressure in the form of Eq. (33), the estimated uncertainty of the mass estimate goes to infinity when the orbit plane, the observer's initial plane-of-sky and the solar plane-of-sky all coincide.

\subsection{Relative strengths}

This section is concluded with some covariance simulations Eq. (9) along true spacecraft trajectories where the observable is 


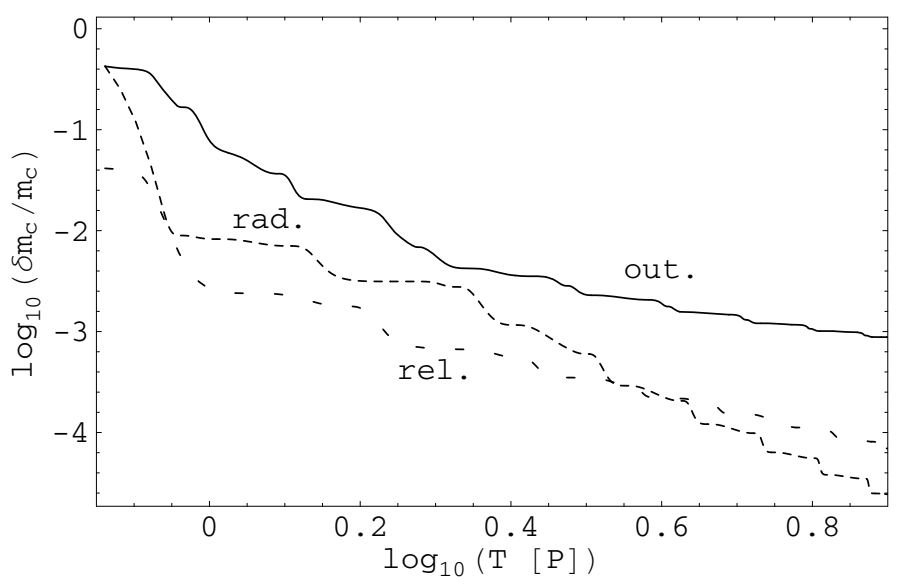

Fig. 5. Estimates of nucleus mass at comet heliocentric distance $R=$ $3.5 \mathrm{AU}$ and cometocentric orbit semi-major axis $a=10 r_{\mathrm{c}}$ are shown as functions of the tracking time $T$ in initial orbit periods $P$. The solid line represents the mass inversion if outgassing pressure is the only effect which lifts the fundamental degeneracy. The corresponding results for relative motion and radiation pressure are represented by the dashed curves.

set to Eq. (4). In addition to the nominal nucleus mass of Eq. (1), the unknown parameters to be estimated are

$$
\begin{aligned}
& \Omega_{0}=f_{0}=\omega_{0}=I_{0}=1, \quad e_{0}=0.2 \\
& p_{0}=8.41 r_{\mathrm{c}}, \quad n_{0}=0.0329 \mathrm{~h}^{-1}, \quad \xi=0.0015 r_{\mathrm{c}} \mathrm{h}^{-2} \mathrm{AU}^{2} \\
& \eta_{b}=0.1, \quad \eta_{s}=1, \quad Q=0.1 r_{\mathrm{c}} \mathrm{h}^{-2}, \quad s=1
\end{aligned}
$$

of Eqs. (29), (30) and (33), where for instance $\Omega_{0} \equiv \Omega\left(t_{0}\right)$ as before. For simplicity $\eta_{d} \equiv 0$ and $\chi_{\odot} \equiv 1$. The a priori parameter uncertainties are set to very large values, or in other words, the parameters are for all practical purposes assumed to be unknown. This is actually not an essential assumption as the estimation filter will erase traces of a priori constraints that are not very precise.

Some results for the mass precision are shown in Fig. 5, where the only information on nucleus mass the filter receives is from outgassing pressure (solid line), radiation pressure (densely dashed) or a relative motion of $\omega_{\oplus}=20 \operatorname{arcsech}^{-1}$ (dashed). That is, our system is defined to be affected by only one of the above perturbations at a time. The cometocentric direction vector of the Sun is defined by the solar latitude $\beta_{\odot}=80^{\circ}$ and longitude $\lambda_{\odot}=160^{\circ}$ relative to the previously defined non-rotating reference system, which at the start of the tracking pass coincides with the observer's plane-of-sky.

In these cases, with a $Q$ which is an order of magnitude lower than what has here been presented as a rough upper limit at comet rendezvous (see Mysen \& Aksnes 2006, and references therein), Eq. (34), outgassing pressure is not essential in the determination of nucleus mass. This is beneficial since it is difficult to know if any aspect of the applied interaction law between the spacecraft and the molecules of the coma is representative before one obtains real data. In a worst case, the variations of the Doppler signal induced by outgassing pressure can only be interpreted as little more than noise, but in the above scenario, a noise which does not dominate the signal.

If, on the other hand, the value of $Q$ is increased an order of magnitude to what is considered as an upper limit at rendezvous, one obtains the results like Fig. 6, where the filter can get a lot

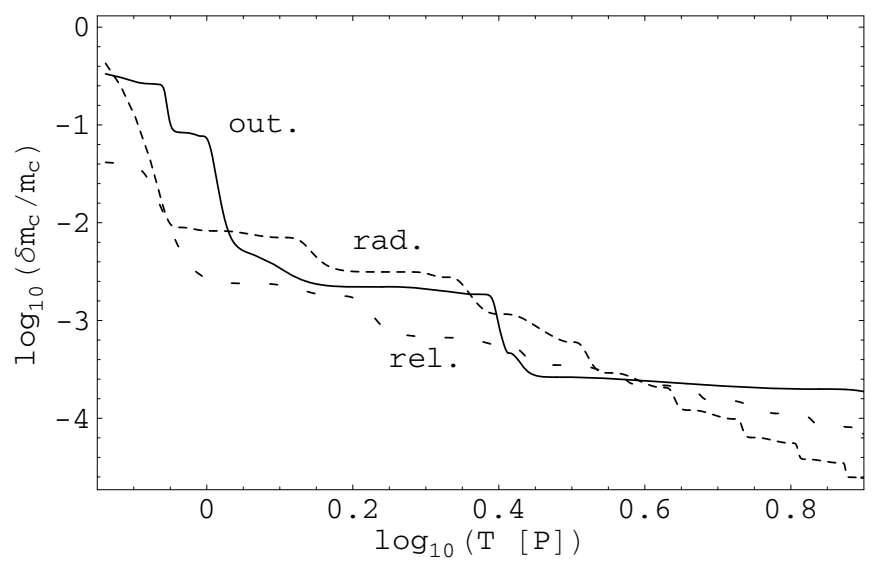

Fig. 6. Precision of the nucleus mass as the Doppler data are collected at an orbit semi-major axis of $a=10 r_{\mathrm{c}}$ as functions of tracking time $T$ in initial orbit periods $P$.

of its information on nucleus mass from the outgassing pressure perturbation.

The same behaviour is also observed for the cometocentric orbit's longitude of ascending node.

At last, it must be mentioned that it will be possible to place quite tight constraints on the radiation pressure parameters during Rosetta's long cruise phase. For instance, covariance simulation runs show that if $\xi$ of the simplified Eq. (29) is known precisely at the start of the tracking pass, the estimate of the nucleus mass will be much improved. However, in order for this improvement to take place, the a priori uncertainty must typically be better than $\delta \xi / \xi \lesssim 0.001$, which seems ambitious to us. Also, upon arrival, the properties of the perturbation may be altered if the spacecraft surfaces are contaminated with dust from an active nucleus, for instance due to CO outgassing (see Mysen \& Aksnes 2006).

\section{Conclusions}

In this paper, the information carried by the Doppler displacements of the radio carrier waves of a probe orbiting a small body has been studied. Fundamental degeneracies of the probe navigation and central body mass determination are rederived, and the related uncertainties are to some extent quantified. Accordingly, navigation on Doppler signal alone is not significantly hampered if the signal is initially lost due to an orbit which at the start of the tracking pass lies in the observer's plane-of-sky. The reason for this is that essential information on the central body mass and navigation parameters are obtained from weak components of the Doppler signal, related to relative motion between observer and the central body.

As a result, it is possible that the variations of the Doppler signal induced by non-gravitational forces can carry more information on the system parameters. On the other hand, if the effect of these non-gravitational perturbations are not well-modelled, which to some extent always is the case for spacecraft, the corresponding information is partly lost. Even further, the induced variations can act as noise which mask components of the Doppler signal which do contain the necessary information for probe navigation and radio science, like those from relative motion. In this paper, it has been demonstrated that this is possible for ESA's Rosetta probe at comet rendezvous in a worst-case outgassing scenario. 
Future extensions of the work in this paper will discuss the extrapolation of the gravity field's details and the coupled rotation parameters of the central body, for which the temporal variations of a possible outgassing pressure field are of importance. A state of the art computer code for coma simulations will be used for this purpose.

Acknowledgements. The first author thanks Susan and Kerry Nock for their kindness and hospitality while visiting Global Aerospace Corporation. The first author would also like to thank John D. Anderson for useful advice and collaboration while at GAC, and Sami W. Asmar for taking care of some important practicalities related to the visit. The authors thank the referees for advice which improved the paper. This work was financed by the Research Council of Norway, projects 170870 and 184686 .

\section{References}

Anderson, J. D., \& Giampieri, G. 1999, Icarus, 138, 309 Crifo, J. F., \& Rodionov, A. V. 1997a, Icarus, 127, 319

Crifo, J. F., \& Rodionov, A. V. 1997b, Icarus, 129, 72
Crifo, J. F., Lukyanov, G. A., Zakharov, V. V., et al. 2004, in The New ROSETTA Targets, ed. L. Colangeli, E. M. Epifani, \& P. Palumbo (Dordrecht: Kluwer), ASSL, 311, 119

Davidsson, B. J. R., \& Gutiérrez, P. J. 2005, Icarus 176, 453

Gelb, A. 1974, Applied Optimal Estimation (Cambridge: M. I. T. Press)

Kossacki, K. J., \& Szutowicz, S. 2006, Planet. Space Sci., 54, 15

Lamy, P. L., Toth, I., Davidsson, B. J. R., et al. 2007, Space Sci. Rev., 128, 23

Moyer, T. D. 2003, Formulation for Observed and Computed Values of Deep Space Network Data Types for Navigation (New Jersey: John Wiley \& Sons)

Mysen, E. 2004, in The New ROSETTA Targets, ed. L. Colangeli, E. M. Epifani, \& P. Palumbo (Dordrecht: Kluwer), ASSL, 311, 211

Mysen, E. 2006, MNRAS, 372, 1345

Mysen, E., \& Aksnes, K. 2006, A\&A, 455, 1143

Pätzold, M., Häusler, B., Aksnes, K., et al., 2007, Space Sci. Rev., 128, 599

Rickman, H., Kamél, L., Festou, M. C., et al. 1987, ESA SP-278, 471

Russell, R. K., \& Thurman, S. W. 1989, An Analytic Development of Orbit Determination for a Distant Planetary Orbiter, AAS/AIAA 89

Tveter, F. T. 1994, Cel. Mech. Dyn. Astron., 60, 409

Valorge, C. 1995, in Spaceflight Dynamics Part I, ed. J.-P. Carrou, CNES, 217

Weeks, C. J. 1995, J. Astronaut. Sci., 43, 327

Wood, L. J. 1986, J. Guid. Cont. Dyn., 9, 485 